\title{
Influence of Growth Break before Capping on Photoluminescence Dynamics of CdSe/ZnSe Self-Assembled Quantum Dots
}

\author{
S. Nowak, J. Suffczyński, M. Goryca, P. Kossacki,
}

$$
\text { J.A. GAJ }
$$

Institute of Experimental Physics, University of Warsaw

Hoża 69, 00-681 Warszawa, Poland

S. LEE

Department of Physics, Korea University, Seoul 136-701, Korea

AND J.K. FURDYNA

Department of Physics, University of Notre Dame

Notre Dame, Indiana 46556, USA

Influence of growth breaks before capping of CdSe self-assembled quantum dot layers on photoluminescence dynamics was examined in three samples. Short (5 s) break resulted only in a small blue shift, caused probably by partial strain relaxation and/or Zn interdiffusion. Long (20 min) break induced a strong broadening and red shift of the spectra, combined with a dramatic slow down of the photoluminescence decays. The main result of the long break was identified as introduction of defects (impurities), which generate local electric fields and act as traps of photogenerated carriers.

PACS numbers: 78.55.Et, 78.67.Hc, 81.07.Ta

\section{Introduction}

Semiconductor quantum dots (QDs) attract attention for their possible application in quantum information technologies. In comparison with classical GaAs-based III-V QD systems, II-VI QDs exhibit more robust excitonic states, and therefore emit light at higher temperatures. Among the physical phenomena occurring in II-VI QDs, exciton dynamics have been extensively studied, e.g., in $\mathrm{CdTe} / \mathrm{ZnTe}[1]$ and CdSe/ZnSe [2] systems. In this work we focus on the influence of the growth break on the exciton dynamics. 


\section{Samples and experiment}

Three samples were grown on $800-1000 \mathrm{~nm}$ thick ZnSe buffers. Self-assembled quantum dots were obtained by deposition of $3 \mathrm{ML}$ (monolayer) of CdSe on the buffer. The CdSe QDs were then capped by $50 \mathrm{~nm}$ of ZnSe. The only difference between the samples was the break time before capping: no break, $5 \mathrm{~s}$ break, or 20 min break, respectively.

The samples were mounted directly on the front surface of a special microscope objective [3] and immersed in superfluid helium in an optical cryostat. They were excited by a laser diode supplying pulses of 100 ps duration at $403 \mathrm{~nm}$ or by a frequency-doubled $\mathrm{Al}_{2} \mathrm{O}_{3}$ :Ti mode-locked laser at the same wavelength. The signal was excited and collected through the microscope objective of numerical aperture 0.7. The microscope assured spatial resolution better than $1 \mu \mathrm{m}$ and allowed us to select single QD emission lines in the low-energy tail of the spectra. Photoluminescence (PL) decays were measured by photon counting, correlated with the laser pulses at different photon energies across the QD ensemble spectrum.

\section{Results and discussion}

\subsection{Photoluminescence characterization}

Photoluminescence spectra of the samples are presented in Fig. 1. With respect to sample $\mathrm{B}$, where no break was applied after the QD layer growth, the PL spectrum of sample C (5 s break) exhibits a blue shift of about $30 \mathrm{meV}$. A blue shift smaller by an order of magnitude was reported under influence of rapid thermal annealing (RTA) of $\mathrm{CdTe} /(\mathrm{Cd}, \mathrm{Mn}) \mathrm{Te}$ quantum wells [4]. Taking into account much stronger strain present usually in quantum dots, we consider a partial strain relaxation as a possible mechanism of the observed blue shift. Another mechanism that may contribute to the observed effect is $\mathrm{Zn}$ diffusion into the QDs. The PL spectrum of sample D is strongly broadened and red-shifted with respect to those of samples B and C. These effects, measured in hundreds of $\mathrm{meV}$, may originate from two sources. The first one is related to ripening processes [5], known to occur in annealed self-assembled QD ensembles, where larger dots grow at the expense of smaller ones. This leads to a decrease in the average confinement energy, and consequently to a red shift. Another contribution to the observed red shift may originate from local electric fields. These fields may be created by impurities and/or defects introduced during the growth break. The observed broadening of the photoluminescence spectrum originates probably from a wide distribution of the confinement energies and local field intensities.

\subsection{Photoluminescence decays}

Decays of photoluminescence have been measured at energies of emitted photons indicated in Fig. 1. In the case of the decay measurements performed at energies from the middle part of the QD ensemble spectrum, emission of multiple QDs contributed to the signal. Two measurements were performed on well 


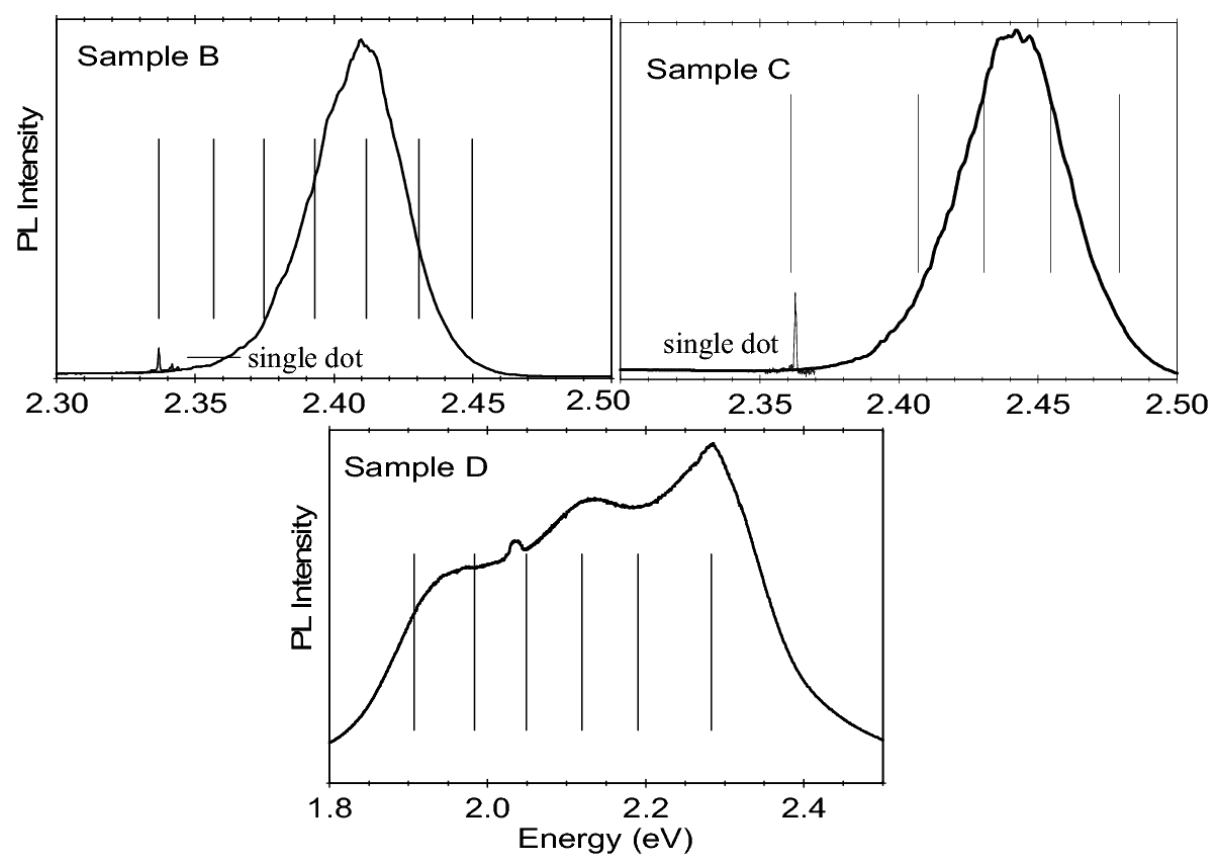

Fig. 1. Photoluminescence spectra od samples B, C and D. Vertical lines indicate energies at which PL decay time measurements were performed.

distinguished individual quantum dot lines coming from the tail of the emission, as shown in Fig. 1. Example decay curves are presented in Fig. 2 together with the fit.

The decay curves are not monoexponential. In samples B and C, biexponential fits gave reliably two decay rates: a fast and a slow one. In case of sample D only the slower decay time was reliably determined, while the decay time of the faster component was uncertain, because of small contribution of the faster component and of a smaller overall PL intensity. In sample B (no break) we observed a strong variation of the excitonic decay rate, which grows with photon energy from about 400 ps at the low energy tail to about 170 ps on the high energy side (Fig. 3). Following previous studies on similar systems [1,2], the observed variation can be explained by tunneling from higher to lower energy dots. However, an alternative explanation may be considered, assuming dominant contribution of highly excited multiexciton fast recombination at the high energy part of the spectrum.

Sample C (5 s break) shows essentially the same behavior, except that the decay time values are shifted in energy together with the PL spectrum. In sample D (20 min break) the decays are generally much slower. Their faster component is of order of a few ns, much more than the corresponding values for the first two samples. A slower component remains in this case in the range between roughly 80 ns and 90 ns and does not exhibit any systematic dependence on the photon 


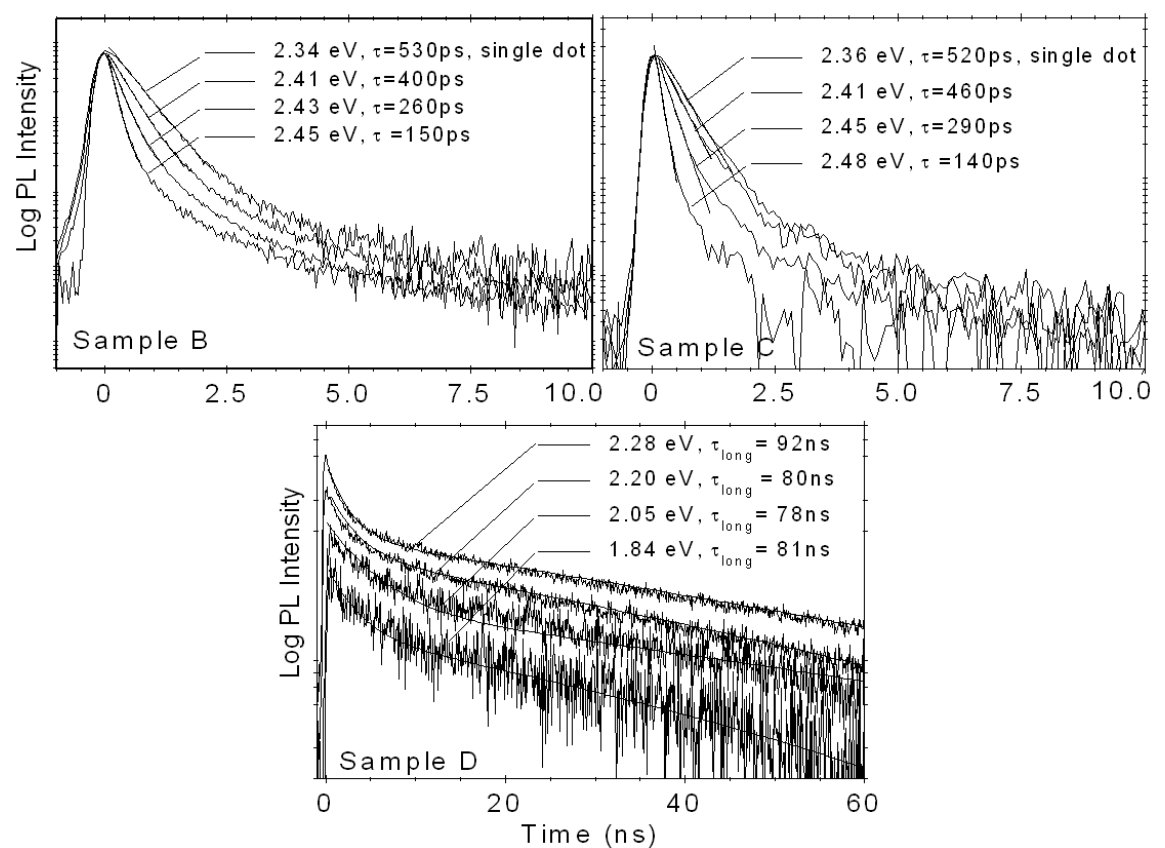

Fig. 2. Examples of excitonic photoluminescence decay curves acquired on samples B, $\mathrm{C}$ and D. Fit of the biexponential function yielded decay time constants. Values of shorter (B and C) and longer (D) decay time constants are indicated on the plots. In the case of sample D decay curves were slightly shifted vertically for the sake of clarity.

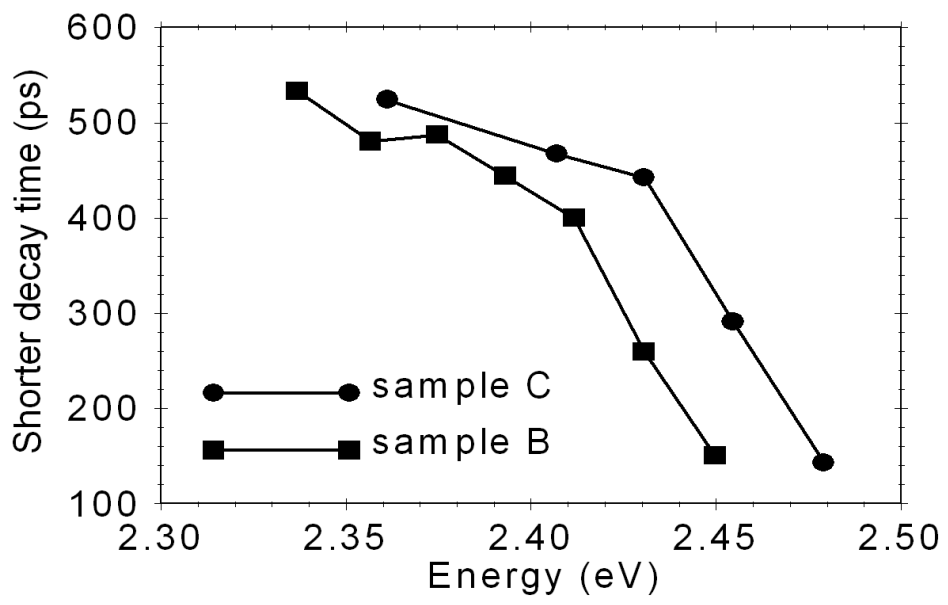

Fig. 3. Variation of the shorter decay time constant with photon energy for samples B and $\mathrm{C}$.

energy. We interpret the faster component as radiative decay, slowed down by local electric fields, which decrease the electron-hole overlap in the QDs. The 
slower component is attributed to a slow release of the photogenerated carriers trapped by centers formed during the 20 min of annealing.

\section{Conclusions}

In conclusion, our measurements point to several processes occurring during the growth break after the formation of CdSe self-assembled QD layers: partial strain relaxation, Zn interdiffusion, and creation of defect centers, which act as carrier traps and as sources of local electric fields.

\section{Acknowledgments}

This work was partially supported by Polish Ministry of Science and Higher Education (projects financed in years 2006-2010), by European project No. MTKD-CT-2005-029671, by the Korea Research Foundation grant KRF-2004-005C00068, and by the National Science Foundations grant DMR06-03762.

\section{References}

[1] K.P. Korona, P. Wojnar, J.A. Gaj, G. Karczewski, J. Kossut, J. Kuhl, Solid State Commun. 133, 369 (2005).

[2] J.H. Park, D.G. Choi, T.K. Lee, Eunsoon Oh, Sanghoon Lee, J.K. Furdyna, Appl. Phys. Lett. 90, 201916 (2007).

[3] J. Jasny, J. Sepioł, Chem. Phys. Lett. 273, 439 (1997).

[4] S. Mac̀kowski, Nguyen The Khoi, P. Kossacki, A. Golnik, J.A. Gaj, A. Lemaitre, C. Testelin, C. Rigaux, G. Karczewski, T. Wojtowicz, J. Kossut, J. Cryst. Growth 184/5, 966 (1998).

[5] S. Lee, I. Daruka, C.S. Kim, A.-L. Barabási, J.L. Merz, J.K. Furdyna, Phys. Rev. Lett. 81, 3479 (1998). 